\title{
Extraskeletal Ewing Sarcoma at Rare Sites with Review of Literature.
}

\author{
Sabah Nayef Nemri', Bouvier Francis Valere D'sa ${ }^{1}$, Sohaila Fatima ${ }^{2}$, Nazima Haider ${ }^{2 *}$ and Nouha Mahmood Alwani ${ }^{1}$ \\ ${ }^{1}$ Aseer Central Hospital, Abha, KSA, Saudi Arabia \\ ${ }^{2}$ King Khalid University, Abha, KSA, Saudi Arabia
}

\begin{abstract}
Ewings sarcoma (ES) is mostly a tumour of children and young adults and is composed of densely distributed, uniform, small to medium sized, round cells with a high nuclear to cytoplasmic ratio. Extraskeletal ES (EES) is a rare presentation and we present 3 cases of EES at uncommon sites involving hard palate, lung and pleura.
\end{abstract}

Keywords: Extraskeletal Ewing sarcoma, Hard Palate, Lung, Pleura, Rare site

\section{Introduction}

Ewings Sarcoma (ES) family of tumors includes the classical bony ES, extraskeletal ES (EES), Askin's tumor (ES of the thoracopulmonary region), and peripheral primitive neuroectodermal tumor (PNET). ${ }^{[1]}$ ES regarded as an undifferentiated type of bone sarcoma of children is now linked with the neoplasm originally described in the soft tissues as PNET because of the presence of the gene fusion resulting from the 11;22 chromosomal translocation. ${ }^{[2]}$ EES is a rare occurrence ${ }^{[3]}$ and significant differences have been noted between EES and classical bony ES. ${ }^{[1]}$ EES most commonly arises from soft tissue of trunk and extremities. ${ }^{[4]}$ We hereby present three cases of EES in very rare locations, two presenting as pleuro-pulmonary masses and the other as hard palate mass.

\section{Case Reports}

Case report 1 A 48 year old male presented with right nasal obstruction and hard palate bulge of 2 months duration. On computed tomography (CT) scan a soft tissue mass centred on the right hard palate measuring $1.8 \times 2.7 \times 3.2 \mathrm{~cm}$ with hard palate bony defect extending superiorly into right nasal cavity and inferiorly into oral cavity with heterogenous enhancement. The paranasal sinuses were within normal limits. (Figure 1A) Excisional biopsy showed nests of atypical cells with hyperchromatic nuclei and scanty cytoplasm at places showing peripheral palisading with abundant fibromyxoid stroma. (Figure $2 A, B)$ Immunohistochemistry (IH) showed strong membranous positivity with CD99, focal positivity for neuron specific enolase (NSE) (Figure $3 A, B$ ) and S100, stromal positivity for vimentin while there was negative reaction with leucocyte common antigen (LCA), CD3, CD20, pancytokeratin, (Figure 4A,B) epithelial membrane antigen(EMA), smooth muscle actin (SMA) and desmin which led to diagnosis of ES/PNET.

Case report 2 A 17-yr-old female presented with difficulty in breathing, left sided chest pain, weight loss of a month duration. CT and Magnetic Resonance Imaging (MRI) showed left hemithoracic huge pleuropulmonary mass measuring $25 \times 16 \mathrm{~cm}$. (Figure 1B) The mass invaded the diaphragm inferiorly and extended to the retroperitoneum as well as the chest wall posteriorly. It invaded the mediastinum and was inseparable from the cardiac silhouette with left pleural effusion. There was a distinct nodule on the apical segment of the right lower lobe without osseous abnormalities. The patient underwent trucut biopsy which revealed small round cells with scanty cytoplasm, round to oval nuclei, fine granular to vesicular chromatin suggestive of malignant small round cell tumour. (Figure 2C) The histological and IH findings were compatible with ES. The tumor cells were diffusely positive for CD 99, vimentin and negative for LCA, synaptophysin, chromogranin, S100, pancytokeratin, desmin, SMA, HMB-45 and TTF1. (Figure 3C,D and Figure 4C, D) Whole body CT and MRI did not reveal any evidence of an occult primary.

Case report 3: A 57 year old non smoker female presented with a two months history of a dry cough and right-sided pleuritic chest pain. Chest radiograph demonstrated a mass in the right lower and middle lobe. (Figure 1C) CT showed a huge heterogenous mass in the right hemithorax $13 \times 8 \times$ $15 \mathrm{~cm}$ in diameter with central heterogeneous enhancement and mass effect over the lung parenchyma and vessels. It showed a wide-base over pleura on the lateral and posterior aspect with minimal amount of pleural effusion mainly in the lower aspect. There was no obvious metastasis in the lung, no lymphadenopathy in the mediastinum and no bony invasion or metastasis. (Figure 1D) The patient underwent CT guided transthoracic biopsy with histological diagnosis of EES. (Figure 2D)

\section{Discussion}

First described in 1921 by American pathologist James Ewing, ES is a malignant small, round, blue cell tumor 


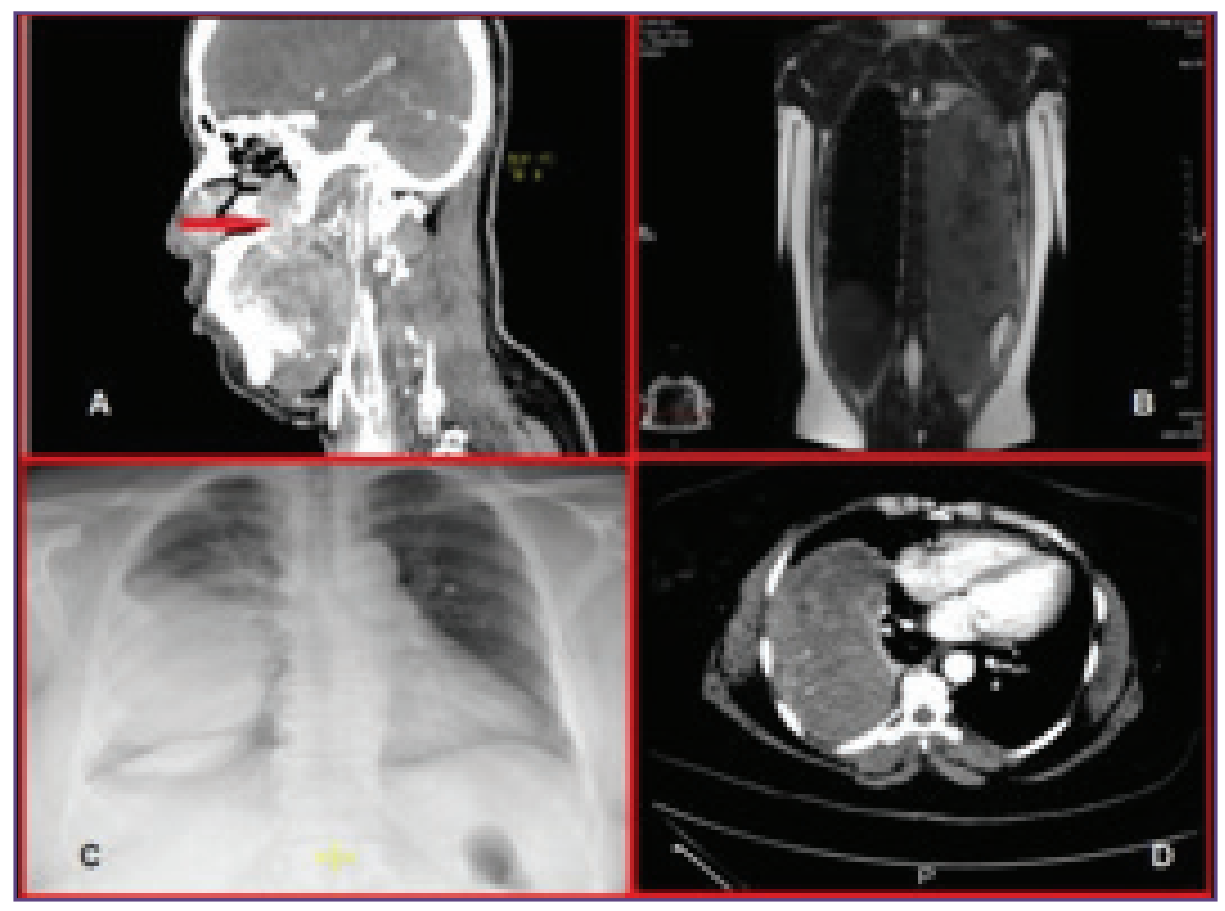

Fig. 1A): CT scan showing a soft tissue mass centred on the right hard palate $(1.8 \times 2.7 \times 3.2 \mathrm{~cm})$ extending superiorly into right nasal cavity and inferiorly into oral cavity. B) MRI showing left pleuropulmonary mass (25 x $16 \mathrm{~cm})$ with mass effect over the mediastinal structures. C) Chest radiograph showing a mass in the right lower and middle lobe. D) CT showing a mass in the right hemithorax. $(13 \times 8 \times 15 \mathrm{~cm})$.

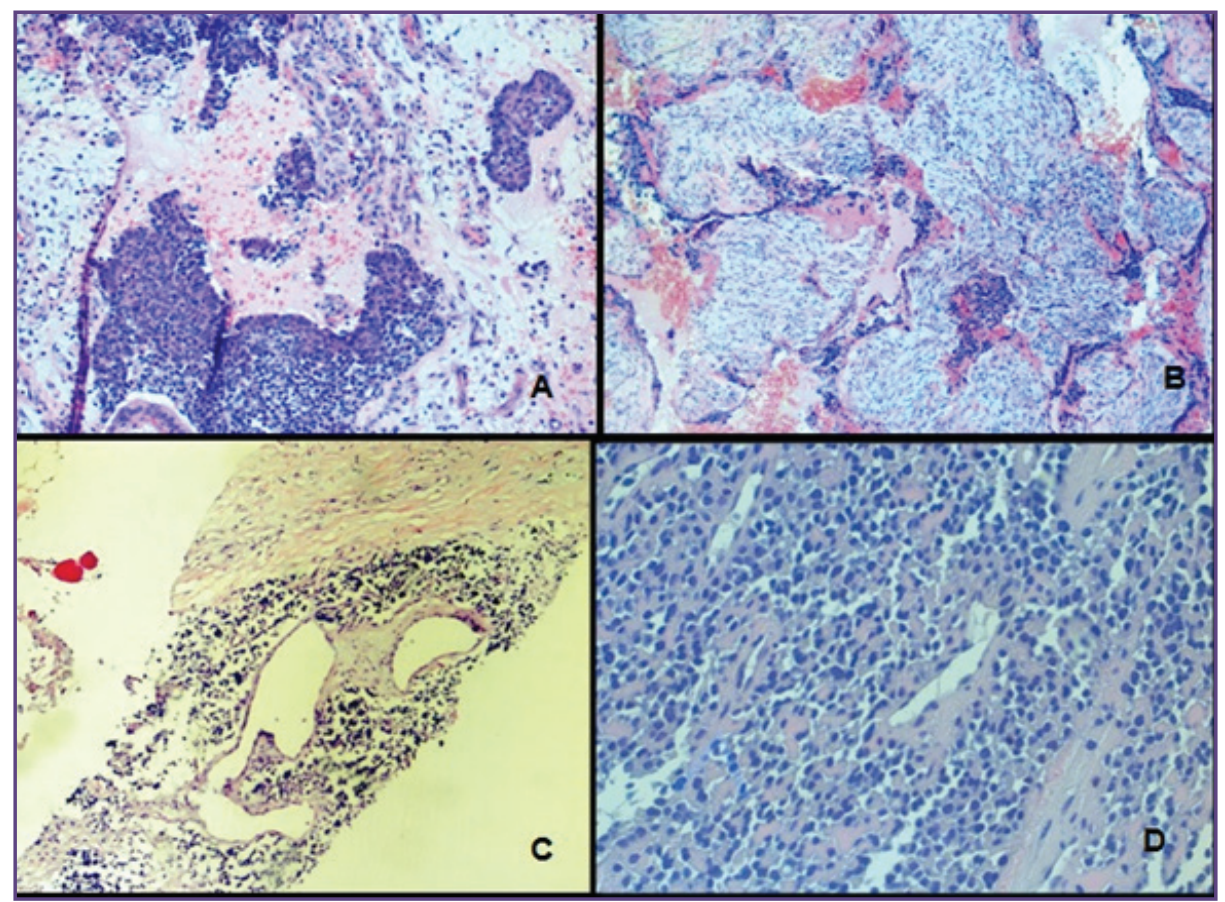

Fig. 2A): Nests of atypical cells with hyperchromatic nucleus and scanty cytoplasm showing peripheral palisading at places. (Hematoxylin and Eosin, 10X) B) Abundant fibromyxoid stroma. (Hematoxylin and Eosin, 10X) C) Small round cells with scanty cytoplasm infiltrating the lung parenchyma. (Hematoxylin and Eosin, 5X) D) Small round cells with scanty cytoplasm, round to oval nuclei, fine granular to vesicular chromatin. (Hematoxylin and Eosin, 40X ). 


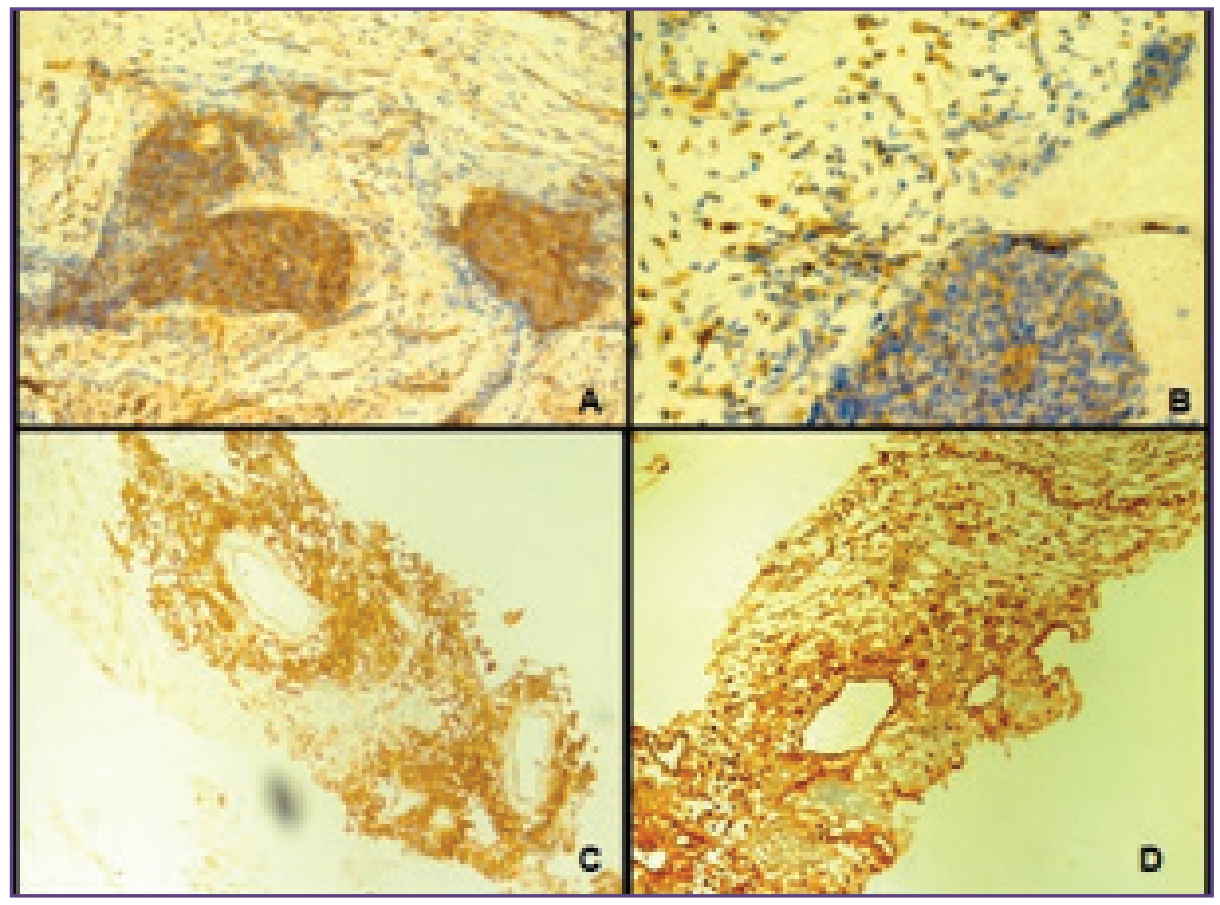

Fig. 3 A): Immunohistochemical study. Nests showing CD99 diffuse positivity. (20X) B) NSE-focal positivity. (20X) C) CD99 diffuse positivity. (10X) 3D) Vimentin-stromal positivity(10X).

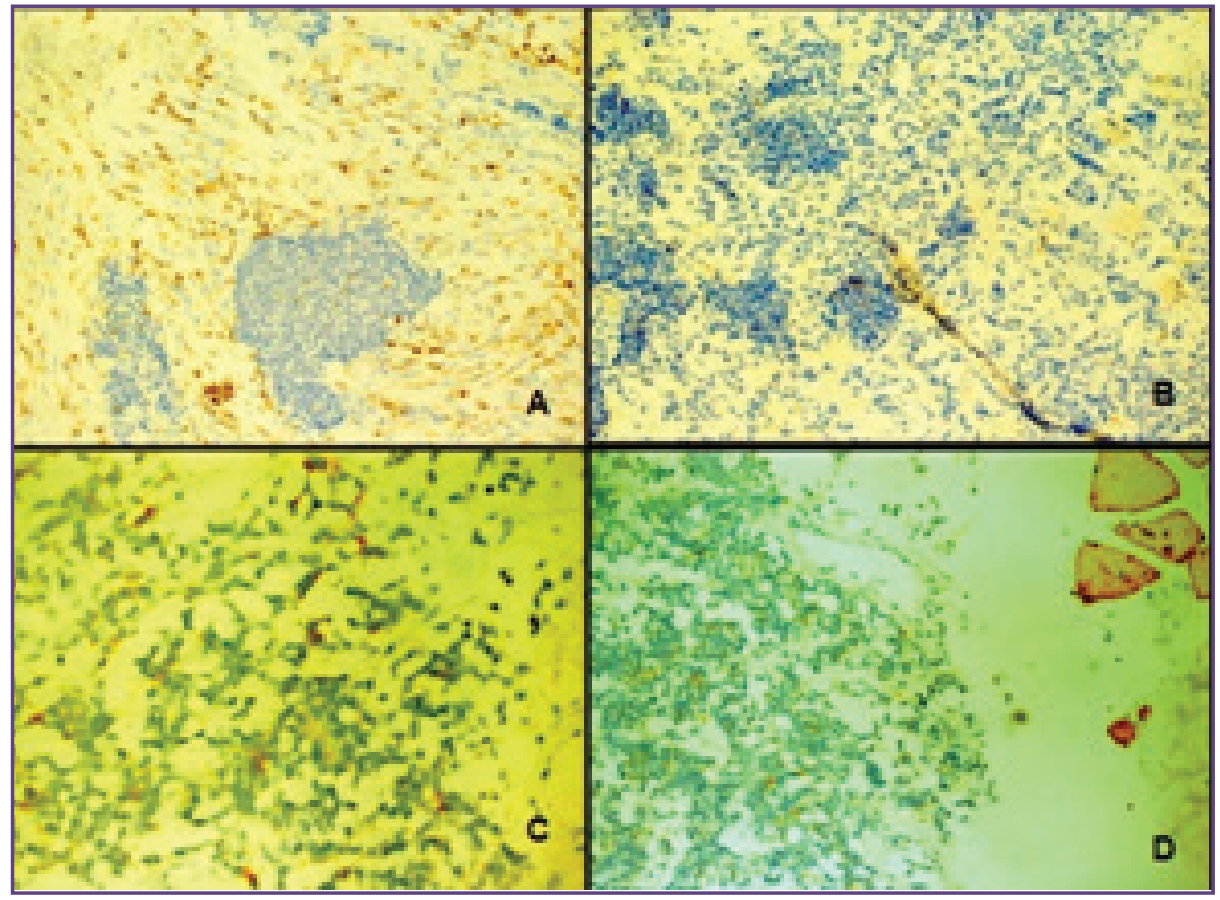

Fig. 4 A): Immunohistochemical study LCA Negative (20X) B) Pancytokeratin Negative (10X) C) Synaptophysin negative (20X) D ) Desmin negative (20X). 
of the bone. ${ }^{[5]}$ It originates from primitive mesenchymal cells (MSCs) of bone marrow or immature reticulum cells. Expression of the EWS-FLI1 fusion gene in MSCs changes cell morphology to resemble ES and induces expression of neuroectodermal markers. ${ }^{[6]}$ Based on this translocation and CD 99 (MIC2 gene product) ES has been grouped into a family of tumors which includes the classical bony ES, EES, Askin's tumor (ES of the thoracopulmonary region), and peripheral primitive neuroectodermal tumor (PNET). ${ }^{[1]}$ EES is a rare entity which can virtually involve any site in the body. It was first described as a soft tissue tumor indistinguishable from ES of bone by light microscopy in 1969. ${ }^{[4]}$

Clinically patients with EES have a higher mean age with a bimodal distribution, more commonly found in those older than 35 and less than 5 years compared with skeletal tumors. Two of our patients are middle aged and one is a teenager. EES shows less male preponderance with most tumors arising in axial locations other than pelvic region. [1] Two out of the three patients are females in our study. Lung, head and neck are rare sites to be involved by EES.

The first case of primary EES of the lung was reported in 1989. ${ }^{[7]}$ Pleural involvement is also very rare which may be primary or secondary due to chest wall ES/PNET. EES is rarely seen in head and neck region; mandible is involved relatively frequently while hard palate and nasal involvement is very rare. ${ }^{[8,9]}$

Imaging of EES shows changes of hemorrhage, cystic change and necrosis but without significant calcification. Xray film shows soft tissue mass without onion skinlike radiation characteristic of ES. CT and MRI show heterogeneously enhancing mass. ${ }^{[10]}$

Microscopically, the typical ES/PNET consists of solid sheets of cells divided into irregular masses by fibrous strands .Cells are small and uniform with indistinct outlines resulting in a syncytial appearance. The nuclei are round with frequent indentations and small nucleoli. There is a well-developed vascular network. Some of the tumor cells may arrange themselves around the vessels in a pseudorosette fashion. A number of histological variants exist- classical, peripheral PNET, atypical, spindle, sclerosing, adamantinoid. ${ }^{[1]}$ Our case presenting with hard palate mass showed nests of atypical cells with peripheral palisading and abundant fibromyxoid stroma. This histologically resembled adamantinoma like ES variant of ES/PNET described by Bridge et al. as an example of "phenotypic drift". ${ }^{[12]}$ Negativity for pancytokeratin in our case helped it to differentiate from adamantinoma like ES. One of possible explanation of finding abundant fibromyxoid stroma and nesting pattern of tumor cells is growth of tumor cells around preexisting fibrovascular structures without destroying them. ${ }^{[9]}$

The differential diagnosis of ES/PNET includes all other 'small round cell tumors' particularly lymphoblastic lymphoma, desmoplastic small cell tumor, embryonal/ alveolar rhabdomyosarcoma, undifferentiated carcinoma, small cell carcinoma-neuroendocrine type, olfactory neuroblastoma, melanoma, synovial sarcoma. CD99/ MIC2 is a cell surface glycoprotein found in virtually all ES and PNET but it is also detected in other small round cell tumors such as T-lymphoblastic lymphoma, poorly differentiated synovial sarcoma, small cell osteosarcoma, rhabdomyosarcoma, desmoplastic small round cell tumor, small cell carcinoma, Merkel cell carcinoma. ${ }^{[13]}$ The panel of other immunostains like vimentin, NSE ,chromogranin, synaptophysin, S100, pancytokeratin, desmin, SMA, LCA, HMB-45, TTF1 helps to reach a conclusive diagnosis as seen in our cases. Ultrastructurally, EES like ES of bone has no collagenous intercellular matrix and cells contain pools of glycogen but do not contain cytoskeleton or intracellular collagen. ${ }^{[14]}$

Treatment involves surgery, chemotherapy and radiation therapy depending on the site, resectability and stage of the tumor. Neoadjuvant chemotherapy with vincristine, doxorubicin, ifosfamide, etoposide, and cyclophosphamide is used before definitive surgery for localized disease and radiation for nonresectable cases. Adults show poor response as compared to children. However, studies have shown complete surgical resection with additional chemotherapy favours good prognosis. ${ }^{[15]}$ EES involving lung and head and neck behave aggressively with surgical resection difficult at times. The most significant prognostic factor is the stage of the disease with metastasis and recurrence carrying a very bad prognosis. ${ }^{[1]}$

In conclusion, primary ES/PNET of lung, pleura, head and neck is uncommon. Small cell morphology leads to wide array of differential diagnosis in these locations with immunohistochemistry playing a key role in reaching the final diagnosis. Early diagnosis helps in appropriate treatment and better prognosis.

\section{References}

1. Applebaum MA, Worch J, Matthay KK, et al. Clinical Features and Outcomes in Patients with Extra Skeletal Ewing Sarcoma. Cancer 2011; 117:3027-3032.

2. Batsakis JG, El-Naggar AK: Ewing's sarcoma and primitive neuroectodermal tumors; cytogenetic cynosures seeking a common histogenesis. Adv Anat Pathol 1997; 4:207-20.

3. El Weshi A, Allam A, Ajarim D, et al. Extraskeletal Ewing's sarcoma family of tumours in adults: analysis of 57 patients from a single institution. Clin Oncol (R Coll Radiol) 2010; 22:374-381. 
4. Tefft M, Vawter GF, Mitus A: Paravertebral "round cell" tumors in children. Radiology 1969;92:1501-1509

5. Ewing J. Classics in oncology. Diffuse endothelioma of bone. Proceedings of the New York Pathological Society, 1921. CA Cancer J Clin. 1972;22:95-98.

6. Lin PP, Wang Y, Lozano G. Mesenchymal Stem Cells and the Origin of Ewing's Sarcoma. Sarcoma 2011:276463. http://dx.doi.org/10.1155/2011/276463

7. Ludwig JA. Ewing sarcoma: historical perspectives, current state-of-the-art, and opportunities for targeted therapy in the future. Curr Opin Oncol 2008; 20:412-418

8. Kissane JM, Askin FB, Nesbit M, Vietti T, Burgert Jr EO, Cangir A, Gehan EA, Perez CA, Pritchard DJ, Tefft M: Sarcomas of bone in childhood. Pathologic aspects. In Glicksmann A, Tefft M (eds): Bone and soft tissue sarcomas. J Natl Cancer Inst Monograph 1981; 56:29-41.

9. Hafezi S, Seethala RR, Edward B. Stelow EB, Mills SE, Leong IT, MacDuff E, Hunt JL, Ordoñez BP, Weinreb I. Ewing's Family of Tumors of the Sinonasal Tract and Maxillary Bone.Head Neck Pathol. 2011; 5(1): 8-16.
10. Javery $\mathrm{O}$, Regan $\mathrm{K} \mathrm{O}$, Jagannathan J, Krajewski $\mathrm{K}$, Giardino A, Ramaiya N H, Kis B. A to Z of Extraskeletal Ewing Sarcoma Family of Tumors in Adults: Imaging Features of Primary Disease, Metastatic Patterns, and Treatment Responses. American Journal of Roentgenology 2011;197:1015-1022. doi10.2214/AJR.11.6667.

11. Pinto A, Dickman P, Parham D. Pathobiologic Markers of the Ewing Sarcoma Family of Tumors:State of the Art and Prediction of Behaviour. Sarcoma, vol. 2011, Article ID 856190, 15 pages, 2011. doi:10.1155/2011/856190

12. Bridge JA, Dembinski A, DeBoer J, Travis J, Neff JR: Clonal chromosomal abnormalities in osteofibrous dysplasia. Cancer 1994,73:1746-52.

13. Weiss SW, Goldblum JR. Enzinger and Weiss's soft tissue tumors. 4th edn. St. Louis: Mosby Inc; 2001. p. 1303.

14. Dickman PS, Triche TJ: Ultrastructural comparison of Ewing's sarcoma of bone with diverse pediatric soft tissue sarcoma resembling Ewing's sarcoma. Lab Invest 1981;44:15-16.

15. Maki RG. Pediatric sarcomas occurring in adults. J Surg Oncol 2008; 97:360-368

*Corresponding author:

Nazima Haider, King Khalid University, Abha, KSA

Phone: 0966-559103778

Email: nazima_haider@yahoo.com

Financial or other Competing Interests: None. 